\title{
Wide Angle Compton Scattering within the SCET factorization framework
}

\author{
Nikolay Kivel ${ }^{\mathrm{a}}$ \\ ${ }^{1}$ Helmholtz Institut Mainz, Johannes Gutenberg-Universität, D-55099 Mainz, Germany
}

\begin{abstract}
Existing data for the electromagnetic proton form factors and for the cross section of the wide angle Compton scattering (WACS) show that the hard two-gluon exchange mechanism (collinear factorization) is still not applicable in the kinematical region where Mandelstam variables $s \sim-t \sim-u$ are about few $\mathrm{GeV}^{2}$. On the other hand these observables can be described in phenomenological models where spectator quarks are soft which assumes a large contribution due to the soft-overlap mechanism. It turns out that the simple QCD factorization picture is not complete and must also include the soft-overlap contribution which can be described as a certain matrix element in the soft collinear effective theory (SCET). Then the leading power contribution to WACS amplitude is described as a sum of the hard- and soft-spectator contributions. The existing experimental data allows one to check certain conclusions based on the assumption about dominant role of the soft-spectator mechanism.
\end{abstract}

\section{Introduction}

The first data for the differential cross section of WACS has already been obtained long time ago [1]. New and more precise measurements were carried out at JLab [2]. Double polarization observables for a polarized photon beam and by measuring the polarization of the recoiling proton were also measured at Jefferson Lab (JLab) [3]. New measurements of various observables at higher energies are planned at the new JLAB $12 \mathrm{GeV}$ facility, see e.g. [4].

The asymptotic limit of the WACS cross section, as predicted by QCD factorization, has been studied in many theoretical works [5-8]. It was found that the leading-twist contribution described by the hard two-gluon exchange between three collinear quarks predicts much smaller cross sections than is observed in experiments. One of the most promising explanations of this problem is that the kinematical region of the existing data is still far away from the asymptotic limit where the hard two-gluon exchange mechanism is predicted to dominate. Hence one needs to develop an alternative theoretical approach which is more suitable for the kinematic range of existing experiments.

Several phenomenological considerations, including the large value of the asymmetry $K_{\mathrm{LL}}$ [3] indicate that the dominant contribution in the relevant kinematic range can be provided by the socalled soft-overlap mechanism. In this case the underlying quark-photon scattering is described by the handbag diagram with one active quark while the other spectator quarks are assumed to be soft.

ae-mail: kivel@kph.uni-mainz.de 
Various models have been considered in order to implement such scattering picture within a theoretical framework: diquarks Refs.[13], GPD-models [9-12] and constituent quarks [14] .

An attempt to develop a systematic approach within the soft collinear effective theory (SCET) framework was discussed in Refs.[15-17]. The description can be considered as a natural extension of the collinear factorization to the case with soft spectators.

In Refs $[15,16]$ the factorization of the three leading power amplitudes has been studied and a phenomenological analysis was made. The three amplitudes describing Compton scattering which involve a nucleon helicity-flip are power suppressed and they were neglected. In Ref.[17] these amplitudes were also included into description, together with all kinematical power corrections. For that purpose the factorization of helicity-flip amplitudes has been discussed assuming that it can be described as a sum of hard- and soft-spectator contributions. Corresponding soft contributions are described as matrix elements of the appropriate subleading SCET operators. Assuming that such soft contributions are dominant we estimate their possible numerical impact on the cross section and asymmetries.

\section{SCET factorization for the WACS amplitudes}

The amplitude of Compton scattering $\gamma(q)+N(p) \rightarrow \gamma\left(q^{\prime}\right)+N\left(p^{\prime}\right)$ can be parametrized as [18]

$$
\begin{aligned}
M^{\gamma p \rightarrow \gamma p} & =-e^{2} \varepsilon^{* \mu}\left(q^{\prime}\right) \varepsilon^{v}(q) \bar{N}\left(p^{\prime}\right) \mathcal{A}^{\mu v} N(p), \\
\mathcal{A}^{\mu v} & =\left\{-\mathcal{T}_{12}^{\mu v}\left(T_{1}+K T_{2}\right)-\mathcal{T}_{34}^{\mu v}\left(T_{3}+K T_{4}\right)+\mathcal{T}_{5}^{\mu v} i \gamma_{5} T_{5}+\mathcal{T}_{6}^{\mu v} i \gamma_{5} K T_{6}\right\},
\end{aligned}
$$

where $e$ denotes the electromagnetic charge of the proton, $N(p)$ is the nucleon spinor. In Eq.(2) we introduced the orthogonal tensor structures

$$
\mathcal{T}_{12}^{\mu \nu}=-\frac{P^{\prime \mu} P^{\prime \nu}}{P^{\prime 2}}, \quad \mathcal{T}_{34}^{\mu \nu}=\frac{N^{\mu} N^{v}}{N^{2}}, \quad \mathcal{T}_{5}^{\mu \nu}=\frac{P^{\prime \mu} N^{v}-P^{\prime v} N^{\mu}}{P^{\prime 2} K^{2}}, \quad \mathcal{T}_{6}^{\mu \nu}=\frac{P^{\prime \mu} N^{v}+P^{\prime v} N^{\mu}}{P^{\prime 2} K^{2}},
$$

with

$$
P=\frac{1}{2}\left(p+p^{\prime}\right), K=\frac{1}{2}\left(q+q^{\prime}\right), P^{\prime}=P-K \frac{(P \cdot K)}{K^{2}}, N_{\mu}=\varepsilon_{\mu \alpha \beta \gamma} P^{\alpha} \frac{1}{2}\left(p-p^{\prime}\right)^{\beta} K^{\gamma} .
$$

The scalar amplitudes $T_{i} \equiv T_{i}(s, t)$ are functions of the Mandelstam variables. In the WACS kinematics $s \sim-t \sim-u \sim Q^{2} \gg \Lambda_{Q C D}$ these amplitudes include various contributions associated with the hard and long distance QCD dynamics. Using the SCET framework one can perform a systematic analysis of the various contributions including one which are associated with the soft spectator scattering (soft-overlap).

It turns out that in the case of nucleon target the soft spectator scattering mechanism is not suppressed comparing the hard spectator one. Therefore the complete factorization description must include both of them. The factorisation description of the soft spectator contribution is more complicate then the hard one because it involves hard and hard-collinear scales. The hard scale appears due to hard subprocesses describing partons with the virtualities of order $Q^{2}$, the hard-collinear scale is associated with the scattering of the hard-collinear particles with the virtualities of order $\Lambda Q$ where we introduced a generic soft scale $\Lambda \sim \Lambda_{Q C D}$. Assuming that hard, hard-collinear and soft scales are well separated $Q^{2} \gg \Lambda Q \gg \Lambda_{Q C D}^{2}$ the factorisation of the hard modes and hard-collinear modes can be carried out. Factorising the hard modes one reduces QCD to the effective theory known as SCET-I which still includes the hard-collinear modes. At the next step one integrate out these modes reducing description to the SCET-II. This effective theory describes the collinear and soft sectors. Corresponding particles have soft virtualities of order $\Lambda^{2}$ and therefore their dynamics is nonperturbative. The 


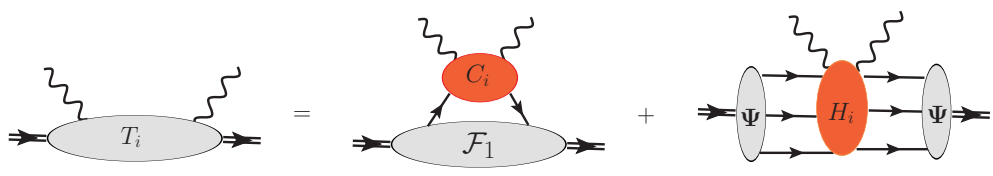

Figure 1. The reduced diagrams describing the factorization formula in Eq.(5).

matrix elements of operators constructed from the SCET-II fields do not depend on the hard scale and must be considered as a nonperturbative.

The kinematical region, where present data have been collected, corresponds to the relatively small value of the hard-collinear scale $\mu_{h c}<1 \mathrm{GeV}$. Therefore in this case one has to stop after factorization of the hard modes. The SCET-I matrix elements in such situation must be also considered as nonpertubative input despite they formally depend on the hard scale $Q$.

In Refs. $[15,16]$ it was shown that the complete leading power factorization formula can be written as

$$
T_{i}(s, t)=C_{i}(s, t) \mathcal{F}_{1}(t)+\boldsymbol{\Psi} * H_{i}(s, t) * \mathbf{\Psi}, \quad i=2,3,4 .
$$

Here the first term on the rhs describes the soft-spectator contribution while the second term corresponds to the well known hard-spectator mechanism. For illustration, in Fig.1 we show the different contributions in Eq.(5) as appropriate reduced diagrams. In the large $Q$ limit both contributions in Eq.(5) behave as $Q^{-6}$ up to logarithmic corrections and give

$$
T_{2,4,6}(s, t) \sim Q^{-6}, \quad Q \rightarrow \infty .
$$

For large values of $Q$ the soft-spectator scattering is strongly suppressed due to the so-called Sudakov logarithms and therefore the hard-spectator contribution becomes dominant. But for moderate values of $Q$ the effect of the Sudakov suppression is still weak therefore the soft-spectator contribution is quite large or even dominant.

The expression in Eq.(5) does not include the amplitudes $T_{1,3,5}$. These amplitudes describe the scattering when the helicity of nucleon is not conserved. Corresponding contribution in the cross section is suppressed by power $O\left(1 / Q^{2}\right)$ comparing the helicity conserving amplitudes. However the factorization of the amplitudes $T_{1,3,5}$ can also be describes in the same way as in Eq.(5) but the soft spectator contribution will be described by the set of the subleading SCET-I operators. More detailed consideration the SCET factorization for the amplitudes $T_{1,3,5}$ is given in Ref.[17].

The asterisks in Eq.(5) denote the convolution with respect to the collinear quark fractions, $\Psi$ denotes the nucleon distribution amplitudes which describe the non-perturbative dynamics in the hardspectator contributions. The hard coefficient functions $H_{i}(s, t)$ in Eq.(5) define the full dependence of the hard-spectator contribution on the Mandelstam variables. The leading-order approximation for these functions are defined by the two-gluon exchange diagrams and therefore they are of order $\alpha_{s}^{2}\left(Q^{2}\right)$.

The hard coefficient functions $C_{2,4,6}$ in the soft spectator term in Eq.(5) can be computed systematically order by order in pQCD. The leading-order expressions read

$$
C_{2}(s, t)=-C_{4}(s, t)=\frac{s-u}{s u}, \quad C_{6}(s, t)=\frac{t}{s u} .
$$

The NLO expressions have also been computed in Ref.[16]. 
The non-perturbative dynamics in the soft-spectator contribution in Eq.(5) is described by the SCET form factor $(\mathrm{FF}) \mathcal{F}_{1}(t)$. In the SCET framework it is defined as

$$
\left\langle p^{\prime}\left|\sum e_{q}^{2}\left\{\bar{\xi}_{n}(0) W_{n} \gamma_{\perp}^{\sigma} W_{\bar{n}}^{\dagger} \xi_{\bar{n}}(0)-\bar{\xi}_{\bar{n}}(0) W_{\bar{n}} \gamma_{\perp}^{\sigma} W_{n}^{\dagger} \xi_{n}(0)\right\}\right| p\right\rangle_{\text {SCET }}=\bar{N}_{n} \gamma_{\perp}^{\sigma} N_{\bar{n}} \mathcal{F}_{1}(t),
$$

where $n$ and $\bar{n}$ denote the auxiliary light-like vectors associated with the nucleon momenta

$$
p=\sqrt{-t} \frac{\bar{n}}{2}+\frac{m_{N}^{2}}{\sqrt{-t}} \frac{n}{2} \simeq \sqrt{-t} \frac{\bar{n}}{2}, \quad p^{\prime}=\frac{m_{N}^{2}}{\sqrt{-t} \frac{\bar{n}}{2}}+\sqrt{-t} \frac{n}{2} \simeq \sqrt{-t} \frac{n}{2},
$$

The fields $\xi_{n}$ and $\xi_{\bar{n}}$ in Eq.(8) denotes the hard-collinear quark fields in SCET-I (with the appropriate flavor) and $W_{n}$ denotes hard-collinear Wilson line

$$
W_{n}(z)=\mathrm{P} \exp \left\{i g \int_{-\infty}^{0} d s \bar{n} \cdot A_{h c}^{(n)}(z+s \bar{n})\right\},
$$

and similarly for the fields with the label $\bar{n}$.

This SCET FF depends only from the large momentum transfer $t$. However this dependence is associated only with the hard-collinear modes which can not be factorized for small values of the hard-collinear scale. One can see from Eq.(5) that the energy $(s)$ dependence is completely defined by the hard coefficient function $C_{i}$ and therefore can be computed in the perturbation theory. SCET analysis allows one to show that at large values of $-t$ one expect that $\mathcal{F}_{1}(t) \sim(-t)^{-2}$. Therefore at large $Q$ the soft spectator term decreases with the same power as the hard spectator contribution.

The FF $\mathcal{F}_{1}(t)$ and the hard coefficient functions $C_{i}$ also depend on the factorization scale $\mu_{F}$ which is not shown for simplicity. This scale defines the separation between the hard and hard-collinear regions. This scale dependence can be computed from the renormalization of the SCET operator in Eq.(8). In the NLO one finds [16]

$$
\mu \frac{d}{d \mu} C_{i}\left(s, t ; \mu^{2}\right)=\frac{\alpha_{s}}{4 \pi} C_{F}\left\{4 \ln \left[-t / \mu^{2}\right]-6+O\left(\alpha_{s}\right)\right\} C_{i}\left(s, t ; \mu^{2}\right),
$$

Solving this equation one can resum the large logarithms to all order in perturbation theory.

\section{Phenomenology}

In order to apply the factorization formula (5) in a phenomenological analysis one must define the unknown nonperturbative form factor $\mathcal{F}_{1}$. This task can only be solved by using a nonperturbative approach within the SCET framework. At present time such approach has not yet developed. Meanwhile a useful phenomenological consideration can be carried out. This is possible due to the universality of the definition of the SCET FF $\mathcal{F}_{1}$ in the factorization approach and due to its specific properties. This one unknown quantity describes the three independent amplitudes $T_{2,4,6}$. Therefore the idea is to re-express this quantity in terms of any one amplitude and then use this expression for the remaining two amplitudes. This allows one to establish the relation between the three amplitudes up to well defined hard-spectator corrections.

In order to be specific let us use Eq.(5) and write for $\mathcal{F}_{1}$ the following expression

$$
\mathcal{F}_{1}(t)=\mathcal{R}(s, t)-\boldsymbol{\Psi} * H_{2}(s, t) * \boldsymbol{\Psi} / C_{2}(s, t) .
$$

Here we define the ratio

$$
\mathcal{R}(s, t)=\frac{T_{2}(s, t)}{C_{2}\left(s, t, \mu^{2}=-t\right)} .
$$


Note that the rhs of Eq.(12) does not depend on the total energy $s$. Substituting this equation in the expressions for the amplitudes $T_{4,6}(5)$ we obtain

$$
T_{i}\left(s^{\prime}, t\right)=C_{i}\left(s^{\prime}, t\right) \mathcal{R}(s, t)+\Psi *\left\{H_{i}\left(s^{\prime}, t\right)-C_{i}\left(s^{\prime}, t\right) \frac{H_{2}(s, t)}{C_{2}(s, t)}\right\} * \boldsymbol{\Psi} .
$$

On the left side of this equation we have a well defined physical amplitude therefore the right side must also be well defined. This means that the potential end-point singularities in the hard-spectator corrections must cancel in the difference on the rhs of Eq.(14). We assume that all the hard coefficient functions in Eq.(14) are defined at $\mu^{2}=-t$. We also used the different values of the total energy $s^{\prime}$ in Eq.(14) in order to stress that the substitution (12) does not depend on the energy $s$.

If the values of the hard-spectator contributions in Eq.(14) are small, then such terms can be neglected and we obtain

$$
T_{i}\left(s^{\prime}, t\right) \simeq C_{i}\left(s^{\prime}, t\right) \mathcal{R}(s, t) \Leftrightarrow \frac{T_{i}\left(s^{\prime}, t\right)}{C_{i}\left(s^{\prime}, t\right)} \simeq \mathcal{R}(s, t) .
$$

Notice that this formula is valid to all orders in $\alpha_{s}$ for the coefficient $C_{i}$ but at order $\alpha_{s}^{2}$ one has to take into account the hard-spectator corrections.

Obviously the choice of the amplitude $T_{i}$ for the definition of the ratio $\mathcal{R}$ in (13) does not play any essential role. In the definition (13) we also used a freedom to fix the factorization scale and chose $\mu^{2}=-t$ as simplest realization.

If the bulk contribution to the amplitudes $T_{i}(s, t)$ is provided by the soft-overlap term then one expects (this is also implied by Eq.(15) ) that the ratio $\mathcal{R}$ depends only very weakly on the energy $s$

$$
\frac{d}{d s} \mathcal{R}(s, t) \simeq 0,
$$

This picture can be verified when comparing with the data. Consider the unpolarised WACS cross section. Using expressions (15) for the amplitudes $T_{2,4,6}$ and neglecting the helicity flip amplitudes $T_{1,3,5} \simeq 0$ and power suppressed terms $\sim m / Q$ we obtain

$$
\frac{d \sigma}{d t}=\frac{\pi \alpha^{2}}{s^{2}}|\mathcal{R}(s, t)|^{2}(-s u)\left(\frac{1}{2}\left|C_{2}(s, t)\right|^{2}+\frac{1}{2}\left|C_{4}(s, t)\right|^{2}+\left|C_{6}(s, t)\right|^{2}\right) .
$$

If the ratio $\mathcal{R}(s, t)$ depends mostly on the momentum transfer $t$ then the energy dependence of the cross section in Eq.(17) is defined only by the hard coefficient functions. Using Eq.(17) one can extract the absolute value $|\mathcal{R}(s, t)|$ and to check the equation Eq.(16) using the experimental data for the cross section. In Fig. 2 we show the results obtained for the ratio $\mathcal{R}$ where we also take into account the power suppressed kinematical corrections. From Fig.2 we see that this approximate scaling behavior is observed in the region where $-u \geq 2.5 \mathrm{GeV}^{2}$. Hence we can adopt this value as a phenomenological lower limit of applicability of the described approach. For smaller values of $u$ the extracted values of $\mathcal{R}$ (shown by the open squares) demonstrate already a clear sensitivity to $s$. Thus one can observe that for $-u=1.3 \mathrm{GeV}^{2}\left(-t=3.7 \mathrm{GeV}^{2}\right)$ the obtained value of $\mathcal{R}$ is about a factor 2 larger than the scaling curve. This observation clearly demonstrates that the given approach cannot describe the cross section data at small values of $u$.

\section{References}

[1] M. A. Shupe, R. H. Milburn, D. J. Quinn, J. P. Rutherfoord, A. R. Stottlemyer, S. S. Hertzbach, R. Kofler and F. D. Lomanno et al., Phys. Rev. D 19 (1979) 1921. 

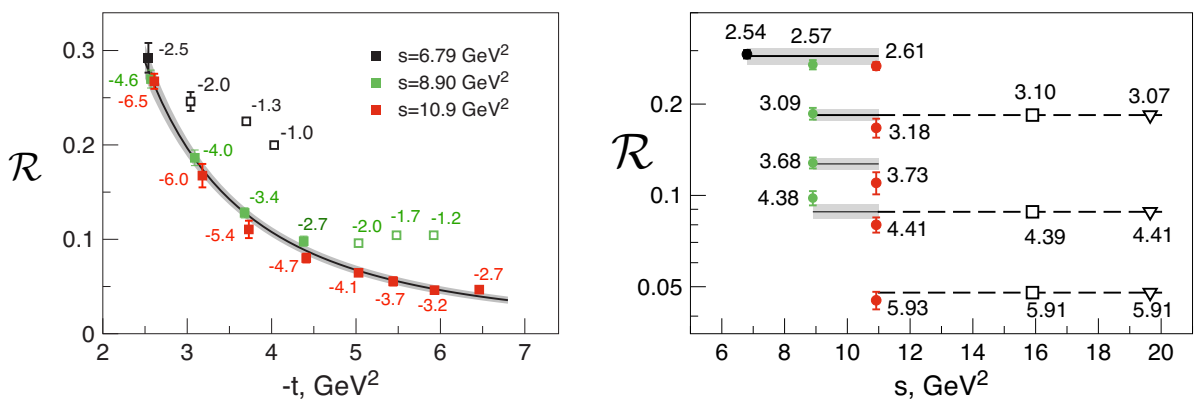

Figure 2. Left: The ratio $\mathcal{R}$ as function of $t$. The corresponding values of $u$ are shown by the numbers next to the symbols. The open squares mark out the points with $-u<2.5 \mathrm{GeV}^{2}$. The solid line represents an empirical fit, the gray bands show the $99 \%$ confidence interval. Right: The ratio $\mathcal{R}$ as a function of energy $s$ at fixed values of $t$ in the region where $-u>2.5 \mathrm{GeV}^{2}$. The corresponding values of $-t$ are shown by the numbers next to the symbols. The open squares and triangles mark out the predicted points which will be checked in cross section measurements at JLab with the $12 \mathrm{GeV}$ beam facility.

[2] A. Danagoulian et al. [Hall A Collaboration], Phys. Rev. Lett. 98 (2007) 152001 [nuclex/0701068 [NUCL-EX]].

[3] D. J. Hamilton et al. [Jefferson Lab Hall A Collaboration], Phys. Rev. Lett. 94 (2005) 242001 [nucl-ex/0410001].

[4] D. Hamilton et al. [Jefferson Lab Hall C Collaboration], E1214003, "Wide-angle Compton Scattering at 8 and $10 \mathrm{GeV}$ Photon Energies".

[5] A. S. Kronfeld and B. Nizic, Phys. Rev. D 44 (1991) 3445 [Erratum-ibid. D 46 (1992) 2272].

[6] M. Vanderhaeghen, P. A. M. Guichon and J. Van de Wiele, Nucl. Phys. A 622 (1997) 144C.

[7] T. C. Brooks and L. J. Dixon, Phys. Rev. D 62 (2000) 114021 [hep-ph/0004143].

[8] R. Thomson, A. Pang and C. -R. Ji, Phys. Rev. D 73 (2006) 054023 [hep-ph/0602164].

[9] A. V. Radyushkin, Phys. Rev. D 58 (1998) 114008 [hep-ph/9803316].

[10] M. Diehl, T. Feldmann, R. Jakob and P. Kroll, Eur. Phys. J. C 8 (1999) 409 [hep-ph/9811253].

[11] M. Diehl, T. Feldmann, R. Jakob and P. Kroll, Phys. Lett. B 460 (1999) 204 [hep-ph/9903268].

[12] M. Diehl, T. Feldmann, H. W. Huang and P. Kroll, Phys. Rev. D 67 (2003) 037502 [hep$\mathrm{ph} / 0212138]$.

[13] P. Kroll, M. Schurmann and W. Schweiger, Int. J. Mod. Phys. A 6 (1991) 4107.

[14] G. A. Miller, Phys. Rev. C 69 (2004) 052201 [nucl-th/0402092].

[15] N. Kivel and M. Vanderhaeghen, JHEP 1304 (2013) 029 [arXiv:1212.0683 [hep-ph]].

[16] N. Kivel and M. Vanderhaeghen, Nucl. Phys. B 883 (2014) 224 [arXiv:1312.5456 [hep-ph]].

[17] N. Kivel and M. Vanderhaeghen, Eur. Phys. J. C 75 (2015) 10, 483 [arXiv:1504.00991 [hep-ph]].

[18] D. Babusci, G. Giordano, A. I. L’vov, G. Matone and A. M. Nathan, Phys. Rev. C 58 (1998) 1013 [hep-ph/9803347]. 